\title{
Intestinal bacteria are in command
}

Two papers in Science present evidence in mice that certain species of intestinal bacteria can drive antitumour immune responses and modulate responses to immune checkpoint blockade.

Sivan et al. investigated the effects of the microbiome on responses to programmed cell death protein 1 (PD1) pathway blockade. B16 mouse melanoma cells engineered to express the SIY antigen (B16.SIY cells) were subcutaneously injected into syngeneic C57BL/6 mice from either Jackson Laboratories (JAX) or Taconic Farms (TAC). Curiously, B16.SIYtumour growth was higher in TAC mice than in JAX mice. The tumours in JAX mice had significantly higher levels of infiltrating SIY-specific $\mathrm{CD}^{+}$ T cells and interferon- $\gamma($ IFN $\gamma)$. These differences were ablated when the JAX and TAC mice were cohoused, which results in their microbiomes becoming similar. Indeed, JAX mouse faecal transplant into TAC mice (by oral gavage) reduced B16.SIY tumour growth and increased tumour-infiltrating SIY-specific $\mathrm{CD}^{+} \mathrm{T}$ cells. TAC mouse faecal material did not significantly increase B16.SIY tumour growth in JAX mice, indicating that the JAX mouse microbiome somehow facilitates $\mathrm{CD}^{+} \mathrm{T}$ cell-mediated antitumour immune responses.

Next, these authors investigated whether the JAX-derived microbiome could modulate responses to PD1 ligand 1 (PDL1) blockade. JAX mice had greater reductions in B16.SIY tumour growth and antitumour immune responses than TAC mice in response to treatment with PDL1 antibodies. Moreover, TAC mice treated with JAX mouse faecal material and PDL1 antibodies had a greater reduction of tumour growth and increased

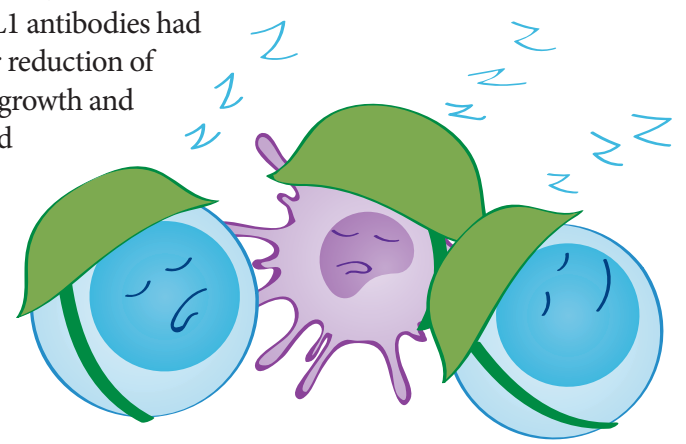

circulating (but not tumourinfiltrating) SIY-specific CD8 ${ }^{+} \mathrm{T}$ cells than treatment with either modality alone. Further investigations revealed that certain Bifidobacterium species were significantly associated with tumour-infiltrating SIY-specific CD8 ${ }^{+}$ T cell-mediated antitumour immune responses and were increased by $>400$-fold in TAC mice receiving JAX mouse faecal material. Oral administration of a mixture of Bifidobacterium species to TAC mice reduced tumour growth and increased tumourinfiltrating SIY-specific $\mathrm{CD}^{+} \mathrm{T}$ cells. Further investigation revealed that signals from Bifidobacterium improve dendritic cell (DC) activation, which leads to improved antigen presentation to, and activation of, $\mathrm{CD} 8^{+} \mathrm{T}$ cells.

Vétizou et al. investigated the effects of the microbiome on responses to cytotoxic $\mathrm{T}$ lymphocyteassociated antigen 4 (CTLA4)

blockade. They implanted MCA205 sarcoma cells expressing the antigen ovalbumin (MCA205.OVA cells) subcutaneously in syngeneic mice that were housed in either specificpathogen free (SPF) or germ-free (GF) conditions. Treatment of these mice bearing established MCA205. OVA tumours with CTLA4 antibodies resulted in reduced tumour growth in SPF mice but had no effect on tumour growth in GF mice. The reduced tumour growth in SPF mice treated with CTLA4 antibodies was prevented by treatment with broad-spectrum antibiotics (ampicillin, colistin and streptomycin; ACS), demonstrating that the microbiome is involved in determining responses to CTLA4 blockade.

intestinal

bacteria

can drive

antitumour

immune

responses

and modulate

responses

to immune

checkpoint

blockade

5

